\title{
DIFFUSE EUV EMISSION FROM CLUSTERS OF GALAXIES
}

\author{
S. BOWYER \\ Space Sciences Laboratory \\ University of California, Berkeley, CA 94720-7450, USA \\ R. LIEU \\ Department of Physics \\ University of Alabama, Huntsville, AL 35988, USA \\ AND \\ J. P. MITTAZ \\ Mullard Space Science Laboratory \\ Holmbury St. Mary, Dorking, Surrey, UK
}

\section{Introduction and Survey of Results}

Diffuse EUV emission has been detected in five clusters of galaxies: Virgo (Lieu et al., 1996a), Coma (Lieu et al., 1996b), Abell 1795, Abell 2199, and Abell 4038. These results were obtained with the Deep Survey Telescope of the Extreme Ultraviolet Explorer (Bowyer and Malina, 1991) using a Lex/B filter which covers the 60 to $170 \AA$ band. The extent of this diffuse emission from these clusters ranges from $20^{\prime}$ to $40^{\prime}$ in diameter. The statistical significance of these results varies from 8 to 50 standard deviations. Some EUV emission would be expected from the low-energy tail of the wellstudied X-ray cluster emission. However, the EUV emission detected is far greater than the expected emission from the X-ray-emitting gas. Marginal signatures of this "soft excess" are often present in the lowest energy resolution band of the ROSAT PSPC where it is typically less than a $20 \%$ effect. In the case of Abell 2199, no low energy excess is present in the ROSAT data. In the data taken with EUVE, however, the excesses range from $70 \%$ to $600 \%$.

Although other suggestions as to the source of this emission have been recently advanced, most workers have interpreted these results in terms of an additional thermal gas component in the cluster. In this work the EUV and X-ray data are first fitted to the know X-ray emitting hot cluster gas. 
The excess EUV emission is then fitted by an additional thermal gas; a much lower temperature gas is required.

\section{Criticisms and Concerns}

We address a number of concerns that have been repeatedly raised regarding the validity of this data, and on alternate suggestions that these data may be the result of detailed characteristics of the Galactic ISM.

A major concern has been that holes in the interstellar medium in our Galaxy would allow excess EUV emission from the X-ray emitting gas through to our location in the Galaxy. However, we have obtained new NRAO twenty-one centimeter data with 21-minute arc resolution, oversampled by a factor of three, for the Virgo, Coma, and Abell 1795 clusters, and similar data with the Effelsberg telescope on the Virgo cluster, with nine arc-minute resolution. All these data show the ISM is smoothly distributed. The distribution of dust in this region was examined with IRAS data, which showed a smooth distribution with four arc-minute resolution. Finally, hydrogen columns have been obtained by absorption in QSO spectra and these give the same hydrogen columns as those obtained by high-resolution radio observations. We conclude that holes in the Galactic ISM cannot be the explanation for these results.

Fabian (1996) has suggested that ionization of neutral helium in the ISM would allow sufficient EUV flux from the low-energy tail of the X-rays to reach Earth and account for the observations. Bowyer et al. (1996) showed that while this effect could work.in principle, the required ionization was vastly at variance with the overall ionization state of helium in the Galaxy.

Concerns have been raised that the effects observed may be artifacts due to the presence of the North Polar Spur in the direction of the Virgo Cluster and Abell 1795 which could produce an uneven background which would distort the observations. Detailed study of the background show this is not a problem, but independent of this analysis, this explanation certainly cannot in any way affect the observations of the Coma Cluster, Abell 2199, and Abell 4038. Hence, this effect cannot be the explanation of what is being observed.

Many critics have questioned whether supposed instabilities in the EUVE detectors may have produced the effects observed. These concerns may be related to the fact that the ROSAT detectors do have variable backgrounds in the lowest energy channels, and the Wide Field Camera underwent substantial changes in its sensitivity over time. However, the EUVE detectors are calibrated every three months through observations of hot white dwarfs. No changes in sensitivity have been observed to a level of a few percent, which is the statistical uncertainty in the observations. A few pixels were 
destroyed when a bright EUV source was observed. However, these dead pixels cover only a few arcseconds and the extended cluster sources typically have twenty to forty arcminute diameters. So this certainly cannot be the explanation for the observations.

It has been suggested that the effect is due to an X-ray leak in the EUVE detector. This concern may arise from the fact that there is an $\mathrm{X}$-ray leak in the Wide Field Camera. However, detailed analyses of the response of the EUVE deep survey telescope shows that this cannot be the case. Independent of the details of this technical analysis, we can eliminate this concern with reference to the data obtained on Abell 1795. In this cluster, the X-ray emission is peaked in the center while the fractional excess EUV emission increases radially outward. If an X-ray leak were producing the effects observed, we would see the maximum pseudo-EUV response in the center of the cluster, but we observe the opposite. This provides confirmation that the EUVE deep-survey telescope detector does not have an X-ray leak which affects these observations.

It has been suggested that an ultraviolet leak in the EUVE deep-survey telescope may be producing these results. This concern may be motivated by the fact that there is a UV leak in the ROSAT PSPC. EUVE, however, does not have a UV leak, and detailed examinations of a large number of stars show that only an eighth-magnitude star or brighter shows any spurious UV signal. There are no O-stars or B-stars even in the general region of any of these clusters.

\section{Theoretical Suggestions}

Most analyses have assumed that the diffuse EUV emission is the result of cooler ( $\sim 1$ million $\mathrm{K})$ gas in these clusters. The problem is that gas at these temperatures are at the peak of the cooling curve and consequently rapidly loses energy. As one example, the cooling of the X-ray gas in the Virgo cluster produces about ten solar masses per year of million-degree gas. However, to produce the observed EUV flux, we need 340 solar masses per year. Hence, if the source of emission is a low-temperature plasma, some mechanism must be present to continually inject energy into this gas.

One suggestion is that this energy could be supplied by energy from the member galaxies of the cluster. The problem with this suggestion is exemplified by Abell 1795 where the total energy requirement is over a Hubble time is $\sim 10^{65}$ ergs, which is 500 times larger than the total energy of all of the supernovae in the central part of the cluster.

Another suggestion (Fabian, 1997) is that the hot X-ray emitting cluster gas contains many cold $\left(10^{4} \mathrm{~K}\right)$ clouds. This increases the EUV emission of the gas by some two orders of magnitude. Unfortunately, cooling is so 
rapid that this process could maintain the existing emission for only one percent of the Hubble time.

Mittaz, et al (1997) have suggested that accretion from the intergalactic medium may be the energy source. This concept works in principle, but for Abell 1795, the total mass accreted over a Hubble time is $4 \times 10^{15}$ solar masses per year. Briel and Henry (1996) obtained this mass for the total virial mass of the cluster, which leaves no room for dark matter, which everyone knows must be present in some exotic form, rather than as a simple plasma.

Several authors (Hwang, 1997; Bowyer and Berghoefer, 1997; and Ensslin and Bierman, 1997) have explored the possibility that high-energy electrons, known to be present from their syncrotron emission, may produce the observed effect via inverse Compton-scattering against the $3^{\circ} \mathrm{K}$ Black Body background.

\section{Conclusions}

We conclude that diffuse EUV emission is present in some clusters of galaxies, and this emission is not due to spurious instrumental effects or effects of the Galactic ISM. Only five clusters have been examined to date, and from this small number it is hard to establish the underlying mechanism producing this emission. More observations of a variety of types of clusters are clearly needed. Unfortunately, for the next several years the Extreme Ultraviolet Explorer may be the only observatory available to study this effect.

\section{References}

S. Bowyer and and R. F. Malina, in EUV Astronomy, ed. R. F. Malina and

S. Bowyer. New York: Pergamon, 1991, 397.

S. Bowyer, M. Lampton, and R. Lieu, Science, 274, 1338, 1996.

S. Bowyer and T. Berghoefer, 1997, in preparation.

U. G. Briel and J. P. Henry, Astrophys. J., 472, 131, 1996.

Ensslin and Bierman, 1997, in preparation.

A. C. Fabian, Science, 271, 1244, 1996.

A. C. Fabian, Science, $\mathbf{2 7 5}, 48,1997$.

C.-Y. Hwang, 1997, in preparation.

R. Lieu, J. P. D. Mittaz, S. Bowyer, F. J. Lockman, C.-Y. Hwang, and J.

H. M. M. Schmitt, Astrophys. J., 458, L5-7, 1996a.

R. Lieu, J. P. D. Mittaz, S. Bowyer, J. O. Breen, J. O. Lockman, E. M. Murphy, and C.-Y. Hwang, Science, 274, 1335, 1996b.

J. P. M. Mittaz, R. Lieu, and F. J. Lockman, Nature, submitted.

C. L. Sarazin, Rev. Mod. Phys., 58, 1, 1986. 